\title{
Antecedentes y fundamentos teóricos de los cursos universitarios de contenido disciplinar con lectura y escritura: un acercamiento al contexto latinoamericano
}

Background and theoretical foundations of university level subject courses that incorporate reading and writing: An overview of the Latin American context

\author{
Eurídice Minerva Ochoa-Villanueva ${ }^{1}$ \\ euridice@iteso.mx \\ Karina Decyre Rengifo-Mattos \\ karinarengifo@iteso.mx \\ ITESO, Universidad Jesuita de Guadalajara, Jalisco, México
}

\section{Resumen:}

Este trabajo presenta un análisis de los antecedentes de los cursos de contenido disciplinar que incorporan a la lectura y la escritura como medios tanto para el aprendizaje de los conocimientos del área, como de la discursividad académica de la disciplina. Asimismo, expone un panorama de los fundamentos teóricos que subyacen a dichos cursos en América Latina y de la manera en que se operan en esta misma región. Se encontró que las teorías a partir de las que se diseñan y ponen en marcha se manifiestan en distintos ámbitos: en la manera en que se conciben el lenguaje, la lectura y la escritura; en la forma en que se entiende su enseñanza; y en el enfoque

\begin{abstract}
:
This article presents a background analysis of subject courses that incorporate reading and writing as a way to learn both subject knowledge and the discursive representation of the discipline. It also provides an overview of the theoretical foundations that underlie these courses in Latin America and how they operate in this region. Findings demonstrate that the theories guiding course design and implementation manifest themselves in at least three different domains: the way language, reading and writing are conceived for the design of these courses; the way the courses are taught; and the way the target students are defined. In addition, three different
\end{abstract}

1 Dirección para correspondencia (correspondence address):

Eurídice Minerva Ochoa Villanueva. Karina Decyre Rengifo Mattos. Av. Periférico Sur Manuel Gómez Morín 8585, Col. ITESO, Tlaquepaque, Jalisco, C.P. 45604 (México). 
desde el que se define a los estudiantes a quienes se dirigen las propuestas. Además, se destacan tres formas de impartirlos: el modelo de profesores asociados, uno disciplinar y un especialista en lengua; el de asesorías, en el que el docente disciplinar recibe asesoría del de lengua; y el de apoyo a programas, en el que el especialista en lengua trabaja con los estudiantes en sesiones paralelas a las del disciplinar. En Latinoamérica, los cursos disciplinares con lectura y escritura como medios para el aprendizaje son heterogéneos no solamente en su formulación de origen, sino en sus fundamentos teóricos y en su operación. Asimismo, presentan diferentes grados de consolidación. Profundizar en su estudio los puede fortalecer, así como enriquecer el campo de conocimiento de la enseñanza de la escritura en la universidad.

\section{Palabras clave:}

Programa de escritura; cursos disciplinares con lectura y escritura; escribir para aprender; escribir en las disciplinas; programas de escritura en Latinoamérica. teaching models can be identified: the associate teacher model that includes both a subject teacher and a writing teacher; the model in which the subject teacher receives advice on how to teach writing; and lastly, the program support model, in which the writing and subject teachers work separately with students in parallel sessions. In Latin America, subject courses that include reading and writing as a means of learning are heterogeneous not only in their formulation, but also in their theoretical foundations and their operationalisation. They are also at different levels of consolidation. Studying these courses in greater depth offers a way not only to strengthen them, but to enrich the field of university-level writing instruction in general.

\section{Key words:}

Writing program; subject courses with reading and writing; WID; WAC; writing programs in Latin America.

\section{Résumé:}

Ce travail fait état d'une analyse des antécédents des cours à contenu disciplinaire incorporant la lecture et l'écriture comme moyens d'apprentissage des connaissances de ce domaine ainsi que du discours académique de la discipline abordée. II fournit, de même, un aperçu des fondements théoriques qui sous-tendent ces cours en Amérique Latine et la manière dont ils opèrent dans cette région. Nous avons trouvé que les théories à partir desquelles ces fondements sont conçus et opèrent se manifestent dans des domaines différents. On le voit à la manière dont le langage, la lecture et l'écriture sont conçus, à la conception de son enseignement, et à l'approche à partir duquel se définissent les propositions aux étudiants. De plus, trois manières différentes de les enseigner sont à retenir: le modèle des professeurs associés: l'un disciplinaire et l'autre spécialiste en langue; le modèle basé sur le conseil, où l'enseignant disciplinaire est conseillé par le professeur de langue; le troisième, le modèle $d^{\prime}$ appui sur des programmes où le spécialiste en langue travaille avec les étudiants lors de sessions parallèles à des sessions disciplinaires. En Amérique Latine, les cours disciplinaires ayant la lecture et l'écriture comme moyens $\mathrm{d}^{\prime}$ enseignement sont hétérogènes, non seulement dans leur formulation de base, mais aussi dans les fondements théoriques et leur gestion. Il faudrait ajouter que ces cours subissent différents degrés de consolidation. Des études plus poussées les rendraient plus solides et l'enseignement de l'écriture à l'université y gagnerait beaucoup.

\section{Mots clés:}

Programme d'écriture; cours disciplinaires avec lecture et écriture; écrire pour apprendre; écrire dans les différentes disciplines; programmes d'écriture en Amérique Latine.

Fecha de recepción: 23-10-2020

Fecha de aceptación: 14-01-2021 


\section{Introducción}

La perspectiva de la lectura y la escritura como procesos que se aprenden de una vez y para siempre en la educación básica es, actualmente, poco aceptada, pues se reconoce que, como actividades socialmente construidas y situadas (Gee, 1991; Barton, 1994; Barton y Hamilton, 2000 y 2005), requieren para su dominio de un cultivo constante e intencionado para que su ejercicio responda a las condiciones de los contextos específicos. Gran parte de esta nueva visión se debe a las discusiones y aportes retomados o generados en el marco de los movimientos Writing Across the Curriculum (WAC) y Writing in the Disciplines (WID), desarroIlados en el contexto de la educación superior anglosajona, que trajeron consigo nuevas formas de abordar la enseñanza de la escritura.

En este entorno se producen los cursos de contenido disciplinar que incorporan a la lectura y la escritura como medios tanto para el aprendizaje de los conocimientos del área, como de la discursividad académica de la disciplina, cuyos principios se encuentran atravesados, en mayor o menor medida, por perspectivas teóricas sobre la naturaleza del lenguaje, de la escritura y de su enseñanza. Es así como son adoptados en Latinoamérica, donde se fortalece la reflexión sobre el papel del estudiante universitario frente al conocimiento de la lectura y escritura especializadas.

El presente trabajo se enmarca en los estudios sobre literacidad académica. Es una recuperación monográfica sobre cursos de contenido disciplinar que integran a la lectura y la escritura como medios para el aprendizaje (CDLEA) en América Latina, y tiene como propósitos identificar los antecedentes que les dieron origen; explorar los fundamentos teóricos que los sustentan; y describir de manera panorámica la forma en que operan en la región. ${ }^{2}$

Estos cursos suelen ser entendidos como programas de escritura o como subprogramas de uno más amplio, aunque en la práctica su implementación, al menos en Latinoamérica, es heterogénea por lo que pueden estar vinculados o no con algún centro de escritura.

2 Agradecemos a los(as) responsables de los programas y centros de escritura que proporcionaron información para este trabajo. Sin su valioso apoyo no habría sido posible integrar la mirada sobre la situación actual, en América Latina, de los CDLEA. 


\section{Contexto de origen de los cursos disciplinares con lectura y escritura como aprendizaje y para el aprendizaje}

Los cursos CDLEA surgen en el contexto de los programas de escritura de algunas universidades norteamericanas en los años 90 (Carlino, 2002). Uno de los antecedentes paradigmáticos es el de las asignaturas de escritura en las disciplinas de la Universidad de Duke, en Carolina del Norte, Estados Unidos, que, al evaluar los resultados poco favorables de su programa de escritura compuesto por un curso de redacción aislado (Carlino, 2002), implementó el aprendizaje de la escritura vinculado al de los contenidos. Estas materias de escritura intensiva consistían en dos asignaturas disciplinares impartidas en cada programa académico, cuya forma de enseñanza tenía como centro la generación de productos escritos, con un seguimiento cercano en su elaboración por parte del profesor, lo que implica retroalimentaciones y revisiones constantes (Carlino, 2002). Tenían como propósito, además de la apropiación de los asuntos disciplinares, orientar sobre convenciones de investigación y de escritura específicas de cada área de conocimiento y de sus formas de indagar y argumentar (Carlino, 2002).

Los antecedentes de estos cursos se encuentran en los movimientos Writing Across the Curriculum (WAC) y Writing in the Discipline (WID) que proponen entrelazar la enseñanza de contenidos de un área del conocimiento con orientaciones para escribir sobre ellos, generalmente desde el enfoque paradigmático de la escritura como proceso y no como producto que, a la par, desarrollaron diversos autores desde distintos enfoques disciplinares, tales como Rohman (1965), Murray (1972), Hayes y Flower (1980), Martin (1985), entre otros.

El movimiento WAC surgió en el Reino Unido a mediados de la década de los sesenta (Marinkovich y Morán, 1998; Gavari y Tenca, 2017), y es ampliamente desarrollado en las universidades norteamericanas (Russel, 1990), en ocasiones en vínculo con los centros y programas de escritura (Gavari y Tenca, 2017). Este enfoque pondera la necesidad de integrar la enseñanza de la lectura y la escritura en los cursos que forman parte de los distintos programas universitarios, desde el presupuesto de que la formación para adquirir conocimientos en un campo de estudios comprende apropiarse no sólo de los conceptos y procedimientos de las disciplinas, sino de su cultura escrita (Russell, 1990; Carlino, 2002, 2004; Bazerman y otros, 2005). 
En este sentido, se concibe las diferentes disciplinas como entramados tanto de conocimientos conceptuales y procedimentales, como de prácticas discursivas y retóricas (Bogel y Hjortshoj, 1984; Carlino, 2002). La premisa según la cual la escritura es una habilidad generalizable que puede ser aprendida independientemente de una matriz disciplinaria es cuestionada por este enfoque (Russell, 1990), pues considera que la concepción de la escritura como un saber técnico separado e independiente del campo de estudios es una de las razones por las que no se han logrado resultados alentadores en lo que respecta a este ámbito en las universidades norteamericanas (Russell, 1990). Por ello, se plantea la necesidad de que la enseñanza de la escritura en la universidad no se circunscriba únicamente a los departamentos de lenguas, sino que se incluya como parte del currículo de los distintos programas y en ella participen quienes tienen el dominio de la discursividad del ámbito, como los departamentos disciplinares o facultades, pues, al ser la escritura una práctica situada, difiere de un campo disciplinar a otro (Russell, 1990; Carlino, 2002).

La idea de transversalizar la enseñanza de la escritura es recurrente a lo largo de la historia de las universidades estadounidenses (Russell, 1990), en las que, como esfuerzos orientados en esa línea, se pusieron en marcha cientos de programas de escritura desde principios del siglo XX en distintas instituciones y diversos niveles (Russell, 1990). Dichos programas son herederos de los enfoques pedagógicos y educativos reformistas que se produjeron en la unión americana durante la primera mitad del siglo XX (Russell, 1990), como la pedagogía progresista de Dewey, Scott y Richards, entre otros, quienes proponen que lenguaje y pensamiento son inseparables y la comunicación es una "transacción social entre socios" (noción de comunicación activa o transaccional) que contribuye a la formación y mejora de sociedades democráticas y justas (Russell, 1990).

La aplicación de la propuesta, sobre todo en las universidades norteamericanas de los años 80, presenta dos variantes teórico-prácticas complementarias que se fueron desarrollando a medida que se reflexionaba sobre lo que ocurría en el aula: 1) el enfoque cognitivo o "escribir para aprender" (writing to learn), cuyo presupuesto de partida consiste en la idea de la escritura como un medio para el aprendizaje; y 2) el retórico o "escribir en las disciplinas" (writing in the disciplines), centrado en la relación entre retórica y formas de saber y pensar en las distintas comunida- 
des disciplinarias, que tiene como propósito introducir a los estudiantes en las diferentes culturas discursivas especializadas (McLeod, 1989).

La perspectiva WAC es antecedente de planteamientos más recientes, como la alfabetización académica, propuesta por Carlino (2003 y 2013b) en el ámbito latinoamericano, o disciplinary literacy en el anglosajón (Airey, 2011).

Cabe señalar que, si bien la propuesta de base de la alfabetización académica comprende los principios del WAC de escribir para aprender y el retórico de la escritura en las disciplinas, así como el de la corresponsabilidad de la enseñanza de la escritura con los programas disciplinares, su aporte más relevante es el énfasis en la visión que se tiene del estudiante, quien al llegar a la universidad no cuenta aún con las prácticas discursivas de su disciplina debido a que éstas forman parte del conocimiento especializado de la misma, y sólo se adquieren al participar en la comunidad disciplinar. Este planteamiento es una declaración de principios sobre la forma de concebir al sujeto que aprende en ese nivel: "[los universitarios] si bien son co-responsables de adquirir los modos disciplinares de leer y escribir no son autónomos para lograrlo" (Carlino, 2013b, p. 367).

\section{La correspondencia del WAC y el WID con algunas perspectivas sobre el lenguaje, la escritura y su enseñanza}

En su evolución, el movimiento WAC se ha ido desarrollando de manera paralela, aunque no necesariamente intencional o expresa, con tres ejes de discusión que sobre la escritura y su enseñanza han formulado diversos estudiosos, de modo que, probablemente, estamos ante un fenómeno como el que describe Chartier (2010) acerca de que no siempre las teorías preceden a las prácticas educativas, y éstas no son forzosamente aplicaciones de las teorías, aunque se suele creer que es así, lo que puede llevar a generar un empalme que confunda dispositivos de investigación con dispositivos de enseñanza.

El primero de los ejes de discusión es el carácter cognitivo-epistémico de la escritura, en tanto instrumento de adquisición y desarrollo de conocimientos (Carlino, 2013a). Olson (1996, 1998 y 2009) y Gottschalk y Hjortshoj (2004) sostienen que, además de incrementar la cantidad de información disponible para una cultura y posibilitar la reproducción 
de saberes, la escritura genera un nivel de representación del lenguaje $y$, por ende, del pensamiento, muy diferente del que se obtiene a través del habla y la acción (Olson, 1996, 1998 y 2009). Al respecto, una consideración importante de Olson (1998) es que la representación del mundo sobre el papel ha modificado la estructura del conocimiento a lo largo de la historia, pues permite el análisis pausado de los contenidos cristalizados o fijados en los textos (ideas, nociones, conceptos, visones de mundo, etc.). Aunado a ello, la actividad cognitiva que se produce durante la escritura permite generar mejores comprensiones sobre los objetos de estudio (Gottschalk y Hjortshoj, 2004) e, incluso, en los escritores experimentados, construir nuevos conocimientos, como reportan Scardamalia y Bereiter (1992). Estos autores encuentran que los escritores maduros refieren el papel fundamental que tiene la escritura en el "desarrollo de sus pensamientos y de su conocimiento" (Scardamalia y Bereiter, 1992, pp. 49-50), caracterizan a la acción de escribir como un proceso de descubrimiento, y mencionan que los textos producidos por ellos influyen in situ sobre su pensamiento, por lo que experimentan una influencia bidireccional entre el pensamiento y lo escrito. En este sentido, la reflexión no sólo es necesaria para la escritura, sino que la escritura tiene efectos en la reflexión (Scardamalia, Bereiter y Steinbach, 1984)

Como ha sido observado por los estudiosos del desarrollo de la cognición, el lenguaje interviene en la formación del pensamiento abstracto (Piaget, 1964/1981, 1980/1989) y favorece el desarrollo cognitivo superior (Vigotsky, 1998). Además, permite de base el desarrollo de nuevos conocimientos, tal como lo explica Halliday (1993), quien menciona que el aprendizaje se produce por medio de la lengua. Al ser el lenguaje la forma prototípica de la semiosis humana, su desarrollo es, al mismo tiempo, el desarrollo del aprendizaje: no puede decirse que el lenguaje sea solamente un dominio del conocimiento, sino que es la condición esencial para el proceso mediante el cual la experiencia deviene en conocimiento (Halliday, 1993).

Aunado a lo anterior, el lenguaje, además de ser el principal medio del pensamiento abstracto y una herramienta de aprendizaje del entor-

3 "Not only is reflection valued as an aid to writing, but writing is valued as an aid to reflection (Murray, 1978; Nystrand y Wiederspiel, 1977; Wason, 1980). Reflection is here viewed, following Piaget (1980), as a dialectical process by which higher-order knowledge is created through the effort to reconcile lower-order elements of knowledge" (Scardamalia, Bereiter y Steinbach, 1984, p. 173). 
no, es una forma de representación de esa percepción (Seiler, 2001) La escritura potencializa este aspecto debido a su rasgo material que permite la visualización y tratamiento de ese pensamiento plasmado en papel. Debido a esto, es plausible afirmar que la escritura posibilita la construcción de objetos de conocimiento, puesto que esta tarea requiere el despliegue de operaciones cognitivas superiores, las que sólo ocurren a través del lenguaje que, en su modalidad escrita, propicia grados complejos de abstracción al descargar a la memoria y facilitar el ir y volver sobre la información. Estos tres aspectos del lenguaje - que interviene en la formación del pensamiento abstracto, que permite el aprendizaje y que construye objetos de conocimiento o del pensamiento- se potencializan en la escritura.

El segundo eje de discusión sobre la escritura y su aprendizaje que tiene puntos en común con el movimiento WAC es el que propone la naturaleza situada de la escritura, en tanto práctica que responde a diversas necesidades comunicativas, contextos diferenciados, comunidades particulares, etc., por lo que la escritura se concibe como una habilidad vinculada a las formas de saber y pensar en las distintas comunidades disciplinares (Russell, 1990). Esta discusión se relaciona con los trabajos clásicos de la sociolingüística de Labov (1972), Gumperz (1982) y Hymes (1972/1996) sobre la variación en el discurso dentro de las comunidades de habla y su uso por grupos específicos, así como las formas en que inciden en la interacción y se correlacionan con el orden social de las comunidades. Los estudios de Labov, por ejemplo, sobre las características lingüísticas del inglés afroamericano, sus convenciones particulares y su estigmatización social, fueron pioneros en este sentido. Vinculadas con esta idea, se encuentran la identificación de Gumperz (1982) sobre el papel que juegan las percepciones de los interlocutores y la influencia del contexto en los eventos de habla, por lo que cada uno es único y diferenciado; así como la noción de competencia comunicativa de Hymes (1972/1996), que se refiere a la necesidad de un dominio no sólo de las reglas del sistema gramatical, sino también de las que se determinan al interior de las diferentes comunidades de habla (reglas de

4 "As stated above, our approach takes the teleonomic character of language as a goaldirected activity into account. In a first approximation it can be said that the goal has two facets: (1) achieving cognition, and (2) representing cognition -both by means of a semiotic system. Language is thus our primary means of thinking and of achieving cognitive insight, and it is at the same time the means for representing such insight" (Seiler, 2001, p. 338). 
uso). Dichas ideas se encuentran en la base de la consideración de la existencia de una cultura escrita propia de las comunidades discursivas disciplinares específicas.

La idea de usos lingüísticos diferenciados o variacionismo hace eco en las propuestas surgidas desde el enfoque sociocultural sobre la literacidad, que consideran que la lectura y la escritura son prácticas sociales que responden a necesidades comunicativas particulares de las comunidades de habla, así como a actitudes, valores y usos que se hacen de ellas en un contexto determinado (Gee, 1991; Barton, 1994; Barton y Hamilton, 2000 y 2005). En este sentido, en la universidad y otros contextos académicos y profesionales, la escritura es una práctica social diferenciada que responde a lo anterior en comunidades disciplinares particulares.

El tercer eje de discusión es el del paradigma de la enseñanza de la escritura como proceso, perspectiva que se sustenta en la afirmación fundamental de Humboldt sobre el lenguaje como actividad (energeia) y no como producto (ergon), frente a la concepción del lenguaje como un objeto formal o abstracto, una "cosa" (Seiler, 2001). Esa visión procesual es ampliamente aceptada y desarrollada por lingüistas de diversas escuelas que la adoptan como base para sus propuestas teóricas, como Hjemslev (1971), Benveniste (1966/2011), Seiler (2001), entre otros.

Las implicaciones de dicho axioma para la formación de una teoría de la escritura se observan en modelos cuyo común denominador es la consideración esencial de la escritura como un proceso compuesto por diferentes etapas. Esta visión se expone, por ejemplo, en los planteamientos de Rohman (1965); en el clásico trabajo de Murray (1972) que establece las distintas fases del proceso para hacer una propuesta didáctica; en modelos cognitivos como el de Hayes y Flower (1980); y en los relacionados con el género, como el de Martin (1985).

En resumen, el movimiento WAC, adoptado en su origen por un $\mathrm{CO}^{-}$ llege en Inglaterra como propuesta para la enseñanza de la escritura, se expandió y convirtió en una corriente que en ocasiones impulsó y en otras dialogó con una serie de discusiones y reflexiones que, a su vez, llevaron a la formulación de planteamientos teóricos en un proceso que puede calificarse, en algunos casos, de inductivo, en tanto la enseñanza concreta no siempre va de la mano de una única propuesta teórico-pedagógica (Chartier, 2010), como se señaló líneas arriba. Lo que ocurre con más frecuencia, aunque con excepciones, es que los 
docentes ponen en marcha estrategias de solución para atender los problemas de la enseñanza de la lectura y la escritura y, posteriormente, los investigadores teorizan sobre los asuntos o identifican los fundamentos teóricos — sobre el lenguaje, la escritura o su enseñanza - que explican las prácticas concretas que tienen lugar en el aula.

En esta dinámica se desarrollan los cursos CDLEA, en interacción con las discusiones referidas. A partir de esto, se adoptan y adaptan en Latinoamérica como una de las estrategias, aunque no la más frecuente, para impulsar el desarrollo de la escritura en los niveles educativos superiores.

\section{Cursos en Latinoamérica}

A continuación, se describen los fundamentos teóricos que sustentan a los cursos de esta naturaleza en la región, así como la forma en la que operan. Para ilustrar lo anterior, y sin intención de hacer una enumeración exhaustiva, se mencionan algunos casos concretos de programas actualmente en marcha en diferentes instituciones de Latinoamérica, con datos recabados entre 2019 y 2020.

\section{Fundamentos teóricos}

Si se explora lo que ocurre en América Latina con los programas de escritura que operan con cursos CDLEA, se observa que los fundamentos teóricos que les subyacen muestran diferentes grados de elaboración, mismos que se identifican en cuatro ámbitos: 1) la concepción de base que tienen del lenguaje; 2) las concepciones particulares que tienen de la lectura y la escritura; 3) la manera en que se posicionan para la enseñanza de las mismas; $y$, 4) la forma en que conciben al estudiante como parte de este entramado. Como puede verse, los últimos tres coinciden con los ejes de discusión de la escritura descritos páginas arriba.

Con respecto al primero de los ámbitos señalados, las perspectivas que predominan entre los programas que muestran expresamente una postura teórica acerca del lenguaje en la región son la de la Lingüística Sistémico Funcional (Halliday y Hasan, 1989 y Halliday, 1993) que, a grandes rasgos, concibe al lenguaje como un sistema semiótico socialmente situado que es el que permite el aprendizaje (Halliday, 1993); y 
el enfoque comunicativo de la lengua, que la concibe como una herramienta que tiene como finalidad la comunicación. Su foco es la competencia comunicativa (Hymes, 1972/1996), que incluye el sistema gramatical y los usos en contextos determinados.

Para el segundo ámbito, las concepciones particulares que se tienen de la lectura y la escritura en la región están asentadas en la visión de la escritura como portadora de un fuerte potencial epistémico (Olson, 1996, 1998 y 2009; Scardamalia, Bereiter y Steinbach, 1984; Scardamalia y Bereiter, 1992; Gottschalk y Hjortshoj, 2004; Carlino, 2013a); y la de la lectura y la escritura como prácticas sociales situadas (Gee, 1991; Barton, 1994; Barton y Hamilton, 2000 y 2005).

En cuanto a las formas de enseñanza de la lectura y la escritura en los cursos disciplinares, en Latinoamérica prepondera la perspectiva que plantea impulsar el aprendizaje de esas habilidades como un proceso, aunque hay dos vertientes: la que pone el foco en los géneros textuales (Rose y Martin, 2012) como realizaciones situadas de escritura, y la que aplica técnicas de enseñanza de segundas lenguas.

La primera vertiente, basada en los planteamientos de la Escuela de Sydney (Rose y Martin, 2012) que, a su vez, se derivan de la Lingüística Sistémica Funcional, aborda el proceso de escritura en dos etapas: la pre-escritura que consiste en analizar el género a desarrollar en un texto modelo, identificar cómo está construido y cómo se conforma cada una de sus partes, para proceder a la segunda etapa Ilamada de escritura, consistente en la redacción del documento propio. Ahora bien, algunos programas en América Latina operan con la versión adaptada por Moyano (2007) que incorpora a la pre-escritura la sub-etapa de planeación, y agrega una tercera fase, la de revisión, donde el docente retroalimenta los documentos construidos.

La segunda vertiente parte de la competencia comunicativa (Hymes, 1972/1996), que incluye el sistema gramatical y los usos en contextos determinados. En congruencia, se deriva una metodología de enseñanza vinculada con la didáctica de las lenguas extranjeras que pone en el centro el desarrollo de la competencia comunicativa.

Con relación a la manera en que los cursos CDLEA conciben al estudiante, el enfoque que sobresale en América Latina es el de la alfabetización académica, que considera que el alumno se incorpora a una comunidad discursiva totalmente nueva (Carlino, 2003) para la que necesita prepararse, lo que exige corresponsabilidad por parte de las institucio- 
nes educativas (Carlino 2013b). En contraste, también hay en la región una perspectiva que estima que los estudiantes Ilegan a la universidad con falencias que hay que remediar.

Es destacable que aquellos programas que formulan expresamente una postura teórica para definir el lenguaje y la escritura han diseñado sus propuestas didácticas en congruencia con esa visión. Ejemplos de lo anterior son el Programa de Lectura y Escritura Académicas de la Universidad de Flores (PROLEA-UFLO), en Buenos Aires, Argentina, cuyos cursos se desarrollaron a partir de los planteamientos de la Lingüística Sistémico Funcional y, en consonancia con esto, se posicionan frente a la enseñanza de la escritura desde la perspectiva de la Escuela de Sydney con un diseño centrado en géneros que facilita la concepción del estudiante como alguien que se acerca por primera vez a esas formas comunicativas, por lo que hay que impulsar su integración a la comunidad discursiva de su disciplina. Por su parte, el Centro de Apoyo para la Lectura, la Oralidad y la Escritura-DIGA del Colegio de Estudios Superiores de Administración, en Bogotá, Colombia, parte del enfoque comunicativo de la lengua (Hymes, 1972/1996), de donde deriva una propuesta que se orienta a desarrollar competencias comunicativas.

\section{Formas de operación}

Algunas de las instituciones que en América Latina cuentan con cursos disciplinares con lectura y escritura como medios para el aprendizaje los consideran como sus programas de escritura; otras, los adscriben a un centro de escritura como parte de las estrategias para impulsar el desarrollo de esas habilidades y, otras, los gestionan de manera paralela y relativamente independiente al centro, es decir, operan un centro y un programa. Esto coincide con lo que señala Molina (2015): "Es muy común que el centro de escritura sea parte integral de un programa de escritura, aunque el uno puede existir aparte o sin el otro" (p. 16).

En cuanto a las formas de impartir este tipo de cursos, predominan tres modelos con distintos énfasis:

1) profesores asociados

2) profesores asesores

3) apoyo a programas 
En el primero, el curso es impartido en colaboración entre un especialista en escritura y un docente disciplinar, quienes se encargan de los géneros y de los contenidos de la disciplina, respectivamente. En este modelo de profesores asociados hay un alto grado de colaboración y negociación entre ambos a fin de establecer los géneros académicos o profesionales a producir por los estudiantes, así como los modos de evaluación de los mismos (Moyano y Giudice, 2016 y Moyano, 2017). Un caso es el del Programa de Desarrollo de Habilidades de Lectura y Escritura Académica a lo largo de la Carrera (PRODEAC), de la Universidad Nacional de General Sarmiento, en Argentina. Otros ejemplos, también en Argentina, son el Programa de Lectura y Escritura Académicas PROLEA, de la Universidad de Flores, donde se imparte cada año y en todas las carreras una materia con este modelo de profesores asociados, y la Subárea de Competencias en Discurso Profesional y Académico, de la Universidad Guillermo Brown, donde se cursan dos asignaturas en el primer cuatrimestre, y una por cada cuatrimestre a lo largo de toda la carrera.

El segundo modelo consiste en asesoría y acompañamiento a los docentes disciplinares para el diseño de unidades de sus cursos con la escritura como herramienta de aprendizaje. Se reúnen el docente disciplinar y el especialista en escritura para diseñar estrategias, actividades, rúbricas u otros recursos que requieran para impulsar la escritura como medio pedagógico. Si el docente lo solicita, se da acompañamiento para la aplicación de instrumentos evaluativos que consideran los aspectos de la escritura, ya sea como procesos o como productos, además de las consultas que consideren necesarias. Ejemplos de este modelo son los Cursos Alfa, del Centro de Recursos para la Escritura Académica Normalista (CREAN) de la Escuela Normal Oficial de Irapuato, México, así como los Cursos E del Centro de Español de la Universidad de los Andes, de Bogotá, Colombia. En ambos programas, cualquier curso de pregrado puede incorporar el componente de escritura académica, y son los docentes disciplinares quienes deciden si integran o no su asignatura. Otro programa es el Ilamado Cursos Asociados del Centro de apoyo a la Lectura, la Oralidad y la Escritura DIGA, del Colegio de Estudios Superiores de Administración-CESA, que se imparten en el primer y segundo semestres del pregrado de Administración de Empresas.

Por su parte, el tercer modelo es, de fondo, un formato de asesorías a estudiantes que se basa en prestarle apoyo a las asignaturas del currí- 
culo de una facultad que tengan un componente importante de lectura y escritura. A cada uno de estos cursos se le asigna un profesor experto en lengua, con un horario alterno al de la clase, para que trabaje con los estudiantes de acuerdo con parámetros convenidos por ambos docentes —el disciplinar y el de escritura-. Así, el profesor de escritura se enfoca en fortalecer aspectos específicos de lecto-escritura de acuerdo con la demanda del profesor disciplinar. Ejemplo del modelo es el programa del Centro de Lectura y Escritura en Español (CELEE) de la Universidad del Rosario, en Colombia, que funciona a partir de una alianza con facultades específicas, para acompañar a los estudiantes de las materias curriculares en sus procesos de lecto-escritura. El programa es semestral y está abierto para todas las carreras que elijan Ilevarlo.

\section{Otros esfuerzos}

En algunos centros y programas de escritura de América Latina se operan alternativas que, sin ser propiamente cursos disciplinares con lectura y escritura como medios para el aprendizaje, buscan incidir en la incorporación de la lecto-escritura en el currículo académico a través de asignaturas de contenido. A los profesores se les ofrecen cursos o asesorías cortas individualizadas — presenciales o en línea-; mientras que, para sus estudiantes, se cuenta con tutorías grupales o intervenciones en el aula (talleres, cursillos, etc.).

Ejemplo de lo anterior son los Microtalleres del ITESO, Universidad Jesuita de Guadalajara, en México, en los que los profesores de las asignaturas disciplinares pueden solicitar apoyo al área de lengua para que un especialista aborde en sus cursos algún género textual o discursivo; ambos docentes acuerdan cómo se caracterizará, desarrollará y evaluará el producto, y participan en una sesión ante el grupo para exponer lo anterior.

Como muestra de talleres o cursos dirigidos a los profesores disciplinares, se encuentran el Diplomado en Lectura y Escritura a través del Currículo en el Nivel Superior que se imparte desde el Programa de Alfabetización Académica de la Pontificia Universidad Católica Madre y Maestra, en la República Dominicana, y la Ruta de Formación Docente Lectura y Escritura Académicas para el Aprendizaje Disciplinar que ofrece el ITESO, Universidad Jesuita de Guadalajara.

Cabe señalar que en la región existe gran diversidad de estrategias 
que se implementan para impulsar las competencias de escritura en la universidad, y hay un continuo entre las que se vinculan a las asignaturas disciplinares - motivo de este artículo- y los cursos generales de escritura. En esa gradación encontramos alternativas que se enfocan en el ejercicio de la escritura a partir de las convenciones discursivas de algunas áreas. Ejemplo de ello es el Programa Lectura y Escritura Académicas (PLEA) de la Pontificia Universidad Católica de Chile, compuesto por cursos curriculares diseñados a partir del análisis de las prácticas y textos (géneros) de las áreas disciplinares en las que se ofrecen.

\section{Conclusiones}

Los cursos de contenido disciplinar que incorporan lectura y escritura como medios para el aprendizaje se desarrollan principalmente sobre la base de dos dimensiones teóricas: la correspondiente a los fundamentos propios sobre concepciones de la lengua/escritura, y la referente a los sustentos pedagógico/didácticos para su enseñanza. En Latinoamérica, desde la década de los 90, cobra relevancia la concepción sobre el estudiante universitario y su papel ante la lecto-escritura especializada, lo que suele formularse expresamente en la implementación de los cursos aquí abordados.

La puesta en marcha de estos cursos disciplinares en la región presenta paralelismos con la trayectoria teórico-práctica del WAC; muestra de lo anterior, entre otras cosas, es el hecho de que en muchos casos los principios teóricos que los sustentan quedan implícitos. A este respecto, debido a que en América Latina los cursos atraviesan por momentos muy diferentes en cuanto a su consolidación, en algunos casos se ha privilegiado la puesta en práctica por encima de la formulación expresa de los referentes teóricos y la propuesta didáctica correspondientes. Lo anterior refleja la necesidad manifiesta de incorporar, de manera transversal en el currículum, estrategias para el desarrollo de habilidades de lectura y escritura.

Si bien este tipo de cursos en su origen son de escritura, en Latinoamérica se observa que algunas instituciones han incorporado la comunicación oral de manera expresa como una instancia de la comunicación académica, es el caso de los programas de la Universidad Guillermo 
Brown, de Argentina, y del Colegio de Estudios Superiores de Administración-CESA, de Colombia.

La instauración de cursos disciplinares en los que se incorporan la lectura, la escritura y, en ocasiones, la comunicación oral, ha ocurrido de manera heterogénea, lo que ha derivado en que haya diversidad en sus formatos, en su operación, en sus componentes y en las perspectivas teóricas que los respaldan. Esto responde a que su puesta en marcha está determinada en gran medida por las condiciones particulares de cada institución, lo que no hace posible una forma única de operarlos.

No obstante, puede afirmarse que cuando un programa o centro instituye cursos de esta naturaleza es porque de alguna manera reconoce el carácter epistémico y situado de la escritura, así como la necesidad de fortalecer su dominio como parte de la formación universitaria. Sin embargo, las propuestas se fortalecerían si definen de manera explícita y clara una postura teórica tanto de su concepción de la lengua como de la escritura, pues aquellos programas que han formulado sus cursos sobre la base de un fundamento teórico determinado cuentan con una ruta didáctica congruente con dicho fundamento lo que, a su vez, permite ocuparse del estudiante desde una perspectiva que empate con esos planteamientos. En resumen, las visiones que se tienen de la lengua y la escritura determinan las formas de enseñarlas y favorecen cierta visión acerca del estudiante.

Seguir investigando los cursos disciplinares con lectura y escritura como medios para el aprendizaje en América Latina permitirá conocer los resultados que han tenido, las dificultades que enfrentan y describir los casos de éxito, para contribuir a la reflexión y, con ello, no sólo a consolidar estos cursos en la región, sino también a enriquecer el campo de estudio de la enseñanza de la lectura y la escritura en la universidad desde una perspectiva latinoamericana.

\section{Referencias}

Airey, J. (2011). Talking about Teaching in English. Swedish university lecturers' experiences of changing their teaching language. Ibérica, 22 (Fall), 35-54.

Barton, D. (1994). Literacy. An introduction to the ecology of written language. Cambridge: Oxford University y Cambridge, Blackwell.

Barton, D. y Hamilton, M. (2000). Literacy practices. En Barton, D., Hamilton, M., e 
Antecedentes y fundamentos teóricos de los cursos universitarios de contenido disciplinar con lectura y escritura: un acercamiento al contexto latinoamericano

Eurídice Minerva Ochoa-Villanueva y Karina Decyre Rengifo-Mattos

Ivanc, R. (eds.) (2000). Situated literacies. Reading and Writing in context (7-15). Nueva York: Routledge.

Barton, D. y Hamilton, M. (2005). Literacy, Reification and the Dynamics of Social Interaction. En D. Barton y K. Tusting (eds.). Beyond Communities of Practice (Learning in doing) (pp. 14-35). Cambridge: Cambridge University Press.

Bazerman, C.; Little, J.; Bethel, L.; Chavkin, T.; Fouquette, D. y Garufis, J. (2005). Reference Guide to Writing across the Curriculum. West Lafayette: Parlor Press.

Benveniste (1966/2011). Problemas de lingüística general. México: Siglo XXI.

Bogel, F. y Hjortshoj, K. (1984). Composition theory and the curriculum. En F. Bogel y K. Gottschalk (eds.) Teaching prose. A guide for writing instructors. New York: Norton, 1-19.

Carlino, P. (2002). Enseñar a escribir en la universidad: cómo lo hacen en Estados Unidos y por qué. Revista Iberoamericana de Educación, 12, 1-16.

Carlino, P. (2003). Alfabetización Académica: Un cambio necesario, algunas alternativas posibles. Educere, 6 (20), 409-420. [fecha de Consulta 16 de febrero de 2020]. ISSN: 1316-4910. Disponible en: https://www.redalyc.org/pdf/356/35662008.pdf

Carlino, P. (2004). Escribir a través del currículum: tres modelos para hacerlo en la universidad. Lectura y Vida. Revista Latinoamericana de Lectura, 1, 16-27.

Carlino, P. (2013a). Escribir en la universidad: Teorías y prácticas en Australia, Canadá y Estados Unidos. Jornadas de Escritura Académica y Profesional. Instituto Tecnológico Autónomo de México, México, DF.

Carlino, P. (2013b). Alfabetización académica diez años después. Revista mexicana de investigación educativa, 18 (57), 355-381.

Chartier, A-M. (2010). Enseñar a leer y escribir, entre teoría y práctica. Conferencia presentada en la V Semana da Educação, da Fundação Victor Civita. São Paulo, Brasil, 20 de octubre de 2010.

Gavari, E. y Tenca, P. (2017). La evolución histórica de los Centros de Escritura Académica. Revista de Educación, 378, 9-29.

Gee, J. (1991). What is literacy? In Candace Mitchel \& Kathleen Weiler (Eds.), Rewriting literacy: Culture and the discourse of the other (pp. 3-11). New York: Bergin \& Garvey.

Gottschalk, K. y Hjortshoj, K. (2004). The Elements of Teaching Writing. Boston: Bedford/ St. Martin's.

Gumperz, J. (1982). Discourse strategies. Cambridge, U.K.: Cambridge University Press.

Halliday, M. y Hasan, R. (1989). Language, context, and text: Aspects of language in a social-semiotic perspective. Oxford: Oxford University Press.

Halliday, M. (1993). Towards a Language-Based Theory of Learning. Linguistics and Education, 5, 93-116.

Hayes, J. R. y Flower, L. S. (1980). "Identifying the Organization of Writing Processes". En L. Gregg y E. Steinberg (Eds.), Cognitive Processesin Writing: An Interdisciplinary Approach, pp. 3-30. Hillsdale, N.J.: Lawrence Erlbaum Associates.

Hjelmslev, L. (1943/1971). Prolegómenos a una teoría del lenguaje [trad. de José Luis Díaz de Llano]. Madrid: Gredos.

Humboldt, W. V. (1836/1990). Sobre la diversidad de la estructura del lenguaje humano y su influencia sobre el desarrollo espiritual de la humanidad. Trad. y pról. de Ana Agud, Barcelona, Anthropos. 
Antecedentes y fundamentos teóricos de los cursos universitarios de contenido disciplinar con lectura y escritura: un acercamiento al contexto latinoamericano Eurídice Minerva Ochoa-Villanueva y Karina Decyre Rengifo-Mattos

Hymes, D. (1972/1996). Acerca de la competencia comunicativa. Forma y Función, 9, 13-37.

Labov, W. (1972). Language in the Inner City: Studies in Black English Vernacular. Philadelphia: The University of Pennsylvania Press.

Marinkovich, J. y Morán, P. (1998). La escritura a través del currículum. Revista signos, 31 (43-44), 165-171.

Martin, J. (1985). Process and Text: Two Aspects of Human Semiosis. En J. D. Benson y W. S. Greaves. (Eds.), Systemic Perspectives on Discourse: selected theoretical papers from the 9th International Systemic Workshop (pp. 248-274). Norwood, N.J., E.U.: Ablex.

McLeod, S. (1989). Writing across the Curriculum: The Second Stage, and beyond. College Composition and Communication, 40(3), 337-343.

Molina, V. (2015). Panorama de los centros y programas de escritura en Latinoamérica. Santiago de Cali, Colombia: Pontificia Universidad Javeriana, Sello Editorial Javeriano.

Moyano, E. (2007). Enseñanza de habilidades discursivas en español en contexto preuniversitario: Una aproximación desde la LSF. Revista Signos, 40 (65), 573-608. https://dx.doi.org/10.4067/S0718-09342007000300009

Moyano, E. y Giudice, J. (2016). Un programa de lectura y escritura universitario: Lineamientos teóricos, características y resultados de aplicación. Revista Grafía, 13 (1), 33-59.

Moyano, E. (2017). Diseño e implementación de programas de lectura y escritura en el nivel universitario: principios y estrategias. Lenguas Modernas, (50), 47-72.

Murray, D. (1972). Teach Writing as Process Not Product. The Leaflet, Fall, 11-14.

Olson, D. (1996). Language and Literacy: what writing does to Language and Mind. Annual Review of Applied Linguistics, 16, 3.

Olson, D. (1998). El mundo sobre el papel. El impacto de la escritura y la lectura en la estructura del conocimiento. Barcelona, Gedisa.

Olson, D. (2009). Language, Literacy and Mind: The Literacy Hypothesis. Psykhe, 18 (1), 3-9.

Piaget, J. (1964/1981). Seis estudios de psicología. México: Seix Barral.

Piaget, J. (1980/1989). La psicogénesis del pensamiento y su significado epistemológico. En Á. Alonso-Cortés (Ed), Lecturas de Lingüística (pp. 263-274). Madrid, España: Cátedra.

Rohman, D. G. (1965). Pre-Writing the Stage of Discovery in the Writing Process. College Composition and Communication, 16 (2), 106-112.

Rose, D. y Martin, J.R. (2012). Learning to Write, Reading to Learn: Genre, knowledge and pedagogy in the Sydney School. Londres: Equinox

Russell, D. (1990). Writing Across the Curriculum in Historical Perspective: Toward a Social Interpretation. College English, 52, 52-73.

Scardamalia, M., Bereiter, C. y Steinbach, R. (1984). Teachability of Reflective Processes in Written Composition. Cognitive Science 8, 173-190.

Scardamalia, M. y Bereiter, C. (1992). Dos modelos explicativos de los procesos de composición escrita. Infancia y aprendizaje, 58, 43-64. 
Antecedentes y fundamentos teóricos de los cursos universitarios de contenido disciplinar con lectura y escritura: un acercamiento al contexto latinoamericano Eurídice Minerva Ochoa-Villanueva y Karina Decyre Rengifo-Mattos

Seiler, H. (2001). The Cologne UNITYP project. En M. Haspelmath, E. König, W. Oesterreicher y W. Raible (Eds.), Language Typology and Language Universals. An International Handbook. Vol. I (pp. 323-344). Berlín, Alemania; New York, USA: Walter de Gruyter.

Vigotsky, L. (1998). Pensamiento y lenguaje. Buenos Aires: Fausto. 
